\title{
Potential functions of microRNAs in starch metabolism and development revealed by miRNA transcriptome profiling of cassava cultivars and their wild progenitor
}

\author{
Xin Chen ${ }^{1,2}$, Jing Xia ${ }^{3,4}$, Zhiqiang Xia ${ }^{1,2}$, Hefang Zhang ${ }^{1,2}$, Changying Zeng ${ }^{1,2}$, Cheng Lu ${ }^{1,2}$, Weixiong Zhang ${ }^{3,4}$ \\ and Wenquan Wang ${ }^{1,2^{*}}$
}

\begin{abstract}
Background: MicroRNAs (miRNAs) are small (approximately 21 nucleotide) non-coding RNAs that are key post-transcriptional gene regulators in eukaryotic organisms. More than 100 cassava miRNAs have been identified in a conservation analysis and a repertoire of cassava miRNAs have also been characterised by next-generation sequencing (NGS) in recent studies. Here, using NGS, we profiled small non-coding RNAs and mRNA genes in two cassava cultivars and their wild progenitor to identify and characterise miRNAs that are potentially involved in plant growth and starch biosynthesis.

Results: Six small RNA and six mRNA libraries from leaves and roots of the two cultivars, KU50 and Arg7, and their wild progenitor, W14, were subjected to NGS. Analysis of the sequencing data revealed 29 conserved miRNA families and 33 new miRNA families. Together, these miRNAs potentially targeted a total of 360 putative target genes. Whereas 16 miRNA families were highly expressed in cultivar leaves, another 13 miRNA families were highly expressed in storage roots of cultivars. Co-expression analysis revealed that the expression level of some targets had negative relationship with their corresponding miRNAs in storage roots and leaves; these targets included MYB33, ARF10, GRF1, RD19, APL2, NF-YA3 and $S P L 2$, which are known to be involved in plant development, starch biosynthesis and response to environmental stimuli.

Conclusion: The identified miRNAs, target mRNAs and target gene ontology annotation all shed light on the possible functions of miRNAs in Manihot species. The differential expression of miRNAs between cultivars and their wild progenitor, together with our analysis of $\mathrm{GO}$ annotation and confirmation of miRNA: target pairs, might provide insight into know the differences between wild progenitor and cultivated cassava.
\end{abstract}

Keywords: MicroRNA, Target Gene, Wild Progenitor, Cassava (Manihot esculenta Crantz)

\section{Background}

MicroRNAs (miRNAs) are small (20-25 nucleotides) non-coding RNAs that have emerged as key players in post-transcriptional gene regulation in plants. They are generated from single-strand RNA precursors that are folded into stem-loop structures, and their abilities to bind to complementary sequences of target mRNAs

\footnotetext{
* Correspondence: wangwenquan@itbb.org.cn

${ }^{1}$ The Institute of Tropical Bioscience and Biotechnology (ITBB), Chinese

Academy of Tropical Agricultural Sciences (CATAS), Haikou 571101, PR China

${ }^{2}$ Key Laboratory of Biology and Genetic Resources of Tropical Crops, Ministry

of Agriculture, Haikou 571101, PR China

Full list of author information is available at the end of the article
}

results in cleavage or degradation of the target mRNAs or suppression of their translation [1-3]. Many studies have revealed important roles of miRNAs in development [4-7], adaptation to biotic and abiotic stresses and resistance to pathogen infection [8-10]. Many miRNAs are also specifically expressed during different stages of plant development and in specific plant organs or tissues [11-15]. For example, miR319 regulates transcription factors of the TCP family, which regulate multiple biological pathways, including hormone biosynthesis and signalling for cell proliferation and differentiation [4]; a set of miRNAs that affect plant hormone homeostasis 
and starch accumulation during grain filling in rice has also been identified [6].

Starch is an insoluble polymer of glucose residues produced by the majority of higher plant species, and is a major storage product of many of the seeds and storage organs produced agriculturally and used for human consumption [16]. Several studies reported that the targets of miRNA involved in the metabolism of carbon, sucrose, starch, lipid, etc. in switch grass [17], potato [18] and rice [6]. For example, miRn45-5p targeted SPS (sucrose-phosphate synthase) gene, miR1436 and miR1862 targeted SS (starch synthase) gene in rice. Cassava (Manihot esculenta Crantz), a woody shrub of the Euphorbiaceae, is one of the most important food crops in the world. It is remarkably productive in terms of its capacity to accumulate biomass and starch, and exhibits extraordinary environmental adaptability. Although about 169 miRNAs and 68 miRNA families have been predicted and characterised in cassava by both a computational approach [19] and small RNA sequencing [20], their potential roles in the production of biomass and starch have never been reported.

We are interested in identifying miRNAs and understanding miRNA functions in photosynthesis and starch accumulation in leaves and storage roots of cassava. Here, we addressed the issues of variation at the level of gene (mRNA) expression and regulation of expressed genes by miRNA in two plant organs (leaf and storage root). The two cassava cultivars (cv. KU50 and Arg7) and their wild progenitor (W14), which was deposited in Chinese Cassava Germplasm Garden and used in this study, have contrasting phenotypes in terms of photosynthetic capacity, starch accumulation and yield of storage roots (Table 1).

\section{Results}

Identification of miRNAs in cultivars and their wild progenitor species

Six small RNA samples from leaves and storage roots of wild progenitor W14 and cultivars KU50 and Arg7 were sequenced using an Illumina Hi-Seq2000 instrument. New miRNAs were identified in cassava in our recent studies. We identified miRNAs that did not have greater than $70 \%$ homology with any other miRNAs in any of the searchable databases, and designated these as new miRNAs. Briefly, we incorporated the sequencing data to conduct a genome-wide search for putative loci with miRNA signatures (see details in Methods), and the expression of the miRNAs detected was quantified based on the genome sequence of AM560 [21], as well as annotation in miRBase (www.mirbase.org). A total of 62 miRNA families were detected, including 19 previously reported ones [22] (Table 2). Among these 62 miRNA families, the presence/absence of members in 51 families was conserved across the three genotypes. Ten new families, i.e., new- $1,-4,-7,-12$ to $-16,-27$ and -28 , were expressed in two cultivars but not in W14. The majority of the detected miRNAs were transcriptionally active in all of the three genotypes, as determined by a normalised number of reads (NNRs). For example, new-11 had more than one thousands of reads in all sequencing libraries. In total, 107 conserved miRNAs belonging to 29 annotated families and 39 new miRNAs from 33 families had detectable expression on the basis of the sequencing data (Additional file 1). Moreover, to confirm the above findings, the expression levels of 36 miRNA families (24 conserved and 12 new) were validated by reverse transcription polymerase chain reaction (Additional file 2).

\section{Targets of cassava miRNAs}

To get insight into or predict the functions of conserved and new miRNAs, a miRNA target search was performed to identify their putative targets (see Methods). A total of 360 loci on cassava unigenes were predicted to be targets of 26 conserved and 27 new miRNA families (Additional file 3), and the remaining 10 miRNA families had no predicted target. The number of targets of each miRNA family ranged from one to 25 (with an average of 9.4) for conserved miRNAs, and from one to seven (with an average of 2.9) for

Table 1 General characteristics of cultivar KU50, Arg7 and wild ancestor W14 used for mining and expression analysis of miRNAs and their target genes

\begin{tabular}{lll}
\hline Characteristic & KU50/Arg7 & W14 \\
\hline Latin name & M. esculenta Crantz & M. esculenta. ssp. flabellifolia \\
Code in Chinese cassava germplasm garden & MS000168/MS000580 & MS000581 \\
Collection site and time & Thailand, 2002/CIAT, Columbia, 2007-01-20 & CIAT, Columbia, 2004-07-30 \\
Number of fruits & few & many \\
Propagation method & stems & seeds \\
Photosynthetic efficiency $\left(\mu \mathrm{mol} / \mathrm{m}^{2} / \mathrm{s}\right)$ & $15.9-38.7$ & $14.6-24.2$ \\
Storage root yield $(\mathrm{kg} / \mathrm{plant} / \mathrm{yr})$ & $3.0-10.0$ & $0.5-1.0$ \\
Starch content of root $(\%)$ & $28.0-32.0$ & $3.0-5.0$
\end{tabular}


Table 2 Discovery of miRNA families and members in wild progenitor W14 and cassava cultivars KU50 and Arg7

\begin{tabular}{|c|c|c|c|c|c|c|}
\hline \multirow[t]{3}{*}{ miRNA family } & \multicolumn{6}{|c|}{ Members } \\
\hline & \multicolumn{2}{|l|}{ W14 } & \multicolumn{2}{|l|}{ Arg7 } & \multicolumn{2}{|c|}{ KU50 } \\
\hline & Leaf & Root & Leaf & $\overline{\text { Root }}$ & Leaf & $\overline{\text { Root }}$ \\
\hline 156 & 11 & 11 & 11 & 11 & 9 & 9 \\
\hline $159,162,393,403,408,2111$ & 2 & 2 & 2 & 2 & 2 & 2 \\
\hline 160 & 7 & 7 & 7 & 7 & 4 & 4 \\
\hline 164 & 3 & 4 & 4 & 4 & 3 & 4 \\
\hline 166 & 8 & 8 & 8 & 8 & 8 & 8 \\
\hline 167 & 6 & 6 & 6 & 6 & 5 & 5 \\
\hline $168,391,397,398,530,827,2950$ & 1 & 1 & 1 & 1 & 1 & 1 \\
\hline 169 & 5 & 5 & 5 & 5 & 4 & 4 \\
\hline 171 & 9 & 9 & 9 & 9 & 9 & 9 \\
\hline 172 & 0 & 2 & 0 & 2 & 0 & 2 \\
\hline 319 & 5 & 6 & 6 & 6 & 6 & 6 \\
\hline 390 & 3 & 3 & 3 & 3 & 2 & 2 \\
\hline 394 & 3 & 3 & 3 & 3 & 3 & 3 \\
\hline 395 & 4 & 4 & 4 & 4 & 4 & 4 \\
\hline 396 & 4 & 4 & 4 & 4 & 3 & 3 \\
\hline 399 & 7 & 7 & 8 & 7 & 4 & 3 \\
\hline 477 & 5 & 5 & 5 & 5 & 4 & 4 \\
\hline 535 & 3 & 3 & 3 & 3 & 1 & 1 \\
\hline New-1, $-4,-7,-27,-28$ & 0 & 0 & 1 & 1 & 1 & 1 \\
\hline New-3, $-25,-34$ & 1 & 1 & 1 & 1 & 0 & 1 \\
\hline New-5, $-6,-26$ & 2 & 2 & 2 & 2 & 2 & 2 \\
\hline $\begin{array}{l}\text { New- } 8 \text { to }-11,-22 \text { to }-24,-30 \\
\text { to }-32\end{array}$ & 1 & 1 & 1 & 1 & 1 & 1 \\
\hline New-12 & 0 & 0 & 1 & 0 & 1 & 1 \\
\hline New-13 to -16 & 0 & 0 & 1 & 1 & 1 & 1 \\
\hline New-17, -33 & 1 & 0 & 1 & 1 & 1 & 1 \\
\hline New-18 & 0 & 1 & 1 & 1 & 1 & 0 \\
\hline New-19 & 0 & 1 & 1 & 1 & 0 & 0 \\
\hline New-20 & 4 & 4 & 4 & 4 & 4 & 4 \\
\hline New-21, -29 & 0 & 1 & 1 & 1 & 1 & 1 \\
\hline
\end{tabular}

Whereas " 0 " indicates that no miRNA is detected in the corresponding genotype/organ, a number from " 1 " to " 11 " indicates the number of detected members of the miRNA family; new miRNAs have names in the format of "new-\#", for example, new-1.

Those miRNAs with names that are underlined were previously reported by Zeng et al. [22].

new miRNAs; the target number varied even within a miRNA family. Among conserved miRNAs, miR162 had one target, whereas six miRNA families (miR156, 164, 172, 319, 396 and 397) had more than 10 targets; among new miRNAs, five miRNA families (new-9, -10 , $-20,-22$ and -26$)$ had one target and four miRNA families (new-1, $-7,-18$ and -19 ) had more than five.
Eight genes were targeted by at least two miRNAs; for example, MYB33 and MYB81 were targeted by miR159 and miR319, and SPL2 and SPL13b were targeted by miR156 and miR535. Many of these targets encode transcription factors; for example, they include the SPL (target of miR156 and miR535), MYB (miR159, miR319 and new-18), HAM3 (miR171), NAC (miR164), ARF (miR160, miR167 and miR169), TCP (miR319), GRF (miR396 and miR477) and SIGMA (new-11) genes. Other miRNA targets that are functional genes included genes that encode the AGPase large subunit 2 (miR394), an F-box protein (miR394), RD19 (miR167), a member of the zinc finger family (miR172), IRX12 (miR397) and two disease resistance proteins (NBS-LRR class; miR396 and -30$)$.

\section{Most miRNAs were highly expressed in the leaves and storage roots of cultivars}

The NNR for each miRNA should be no less than 50 , and if the NNR of a miRNA is less than 50 then it is considered to be silent (not expressed at a detectable level). Whereas 34 miRNAs (24 families) displayed a two-fold difference in their expression level between the cultivars and W14 (Table 3), 29 miRNAs (18 families) were highly expressed in the leaves of cultivars, including miR169e, miR2950, miR319cd, miR391, miR393ab, miR394abc, miR395abcd, miR399d, miR535b, new-4, new-6ab, new-12, new-13, new-15, new-17, new-28, new-29 and new-32. On the other hand, six miRNA families were more highly expressed in leaves of W14 than in cultivars, including miR156ijk, miR396c, miR477e, new-11, new-30 and new-34.

Furthermore, a total of 44 miRNAs (23 families) were differentially expressed in storage roots between cultivars and the wild progenitor. Thirteen miRNA families were highly expressed in storage root of cultivars; these included miR156ijk, miR167acdef, miR169e, miR2111ab, miR397, miR399d, miR477cd, new-6ab, new-9, new-15, new-17, new-28 and new-29. Meanwhile, ten miRNA families were more highly expressed in the storage roots of wild progenitor W14; these included miR168, miR171abcdefghi, miR2950, miR393ab, miR396c, new-3, new-8, new-11 and new-34 (Table 3).

\section{Confirmation of miRNA:target pairs by RLM-RACE}

Binding of a miRNA to the complementary sequence in target mRNA leads to mRNA cleavage. The cleavage sites located at its complementary region (CR) are direct evidence of RNA-induced silencing complex (RISC)-mediated slicing of the target mRNA. Fifteen miRNA:target pairs were selected for slicing analysis using RNA ligasemediated rapid amplification of the 5' cDNA ends technique (RLM-RACE). Of these, nine miRNAs (related to 12 pairs) exhibited differential expression between the cultivars 
Table 3 miRNAs that are differentially expressed between cultivars and their wild progenitor

\begin{tabular}{lll}
\hline Organ & Highly expressed in cultivars & Highly expressed in the wild progenitor \\
\hline Leaf & miR169e, miR2950, miR319cd, miR391, miR393ab, miR394abc, & miR156ijk, miR396c, miR477e, new-11, new-30, new-34 \\
& miR395abcd, miR399d, miR535b, new-4, new-6ab, new-12, & \\
& new-13, new-15, new-17, new-28, new-29, new-32 & \\
Root & $\begin{array}{l}\text { miR156ijk, miR167acdef, miR169e, miR2111ab, miR397, miR399d, } \\
\text { miR477cd, new-6ab, new-9, new-15, new-17, new-28, new-29 }\end{array}$ & miR168, miR171abcdefghi, miR2950, miR393ab, miR396c, \\
& new-3, new-8, new-11, new-34 \\
\hline
\end{tabular}

and W14. The corresponding primer sets are listed in Additional file 4. Cloning and sequencing of the PCR amplicons of remnant mRNAs enabled determination of the nucleotide position when a slicing event occurred. Finally, slicing events occurred in 14 of 15 miRNA:target pairs. The cleavage sites of eight pairs were at the 10th, 11th and 12th nucleotides in the CR (Table 4 and Figure 1). miR394 sliced two targets at different sites with different efficiencies: two slicing events at the 10th nucleotide in the CR and seven events downstream of the CR for F-box mRNA; there were also four slicing events at the 2nd and 14th nucleotides in CR and 14 slicing events upstream or downstream of $\mathrm{CR}$ occurred for ADP-glucose pyrophosphorylase large subunit 2 (APL2) mRNA.

Similarly, other miRNAs sliced their targets at the CR. For instance, miR160 sliced ARF10, with ten cleavage sites at the 11th nucleotide in the CR, and miR169 sliced $N F-Y A 3$, with six cleavage events at the CR. In addition, miR396 cleaved its target GRF1 at the classic 11th site. For the remaining five pairs, cleavage sites could not be detected at the CR region, whereas they were detected up/downstream of this region. The cleavage sites of miR398:Dir-like and miR477:CLB were at the +8th and +37 th nucleotides upstream of the $C R$, respectively. Finally, the cleavage sites of miR166:GH17 and new-12: EDR2 were all downstream of their CRs. Taken together, these findings indicated that many cleavage sites were not positioned at the $\mathrm{CR}$ or were even far away from it. Accordingly, these remnant mRNAs might have been randomly degraded mRNAs.

\section{Co-expression analysis of the miRNAs and their targets in leaf and storage root of cassava cultivars and their wild progenitor}

To further study the relationship between miRNAs and their targets, RNA-seq transcription profiling was performed to assay the expression of targets in leaf and storage root among these three Manihot genotypes. Co-expression analysis involved 32 miRNA families highly expressed in leaf or root $(61$ miRNAs $)$ and their 87 corresponding targets. In general, the expression levels of 28 targets were negatively correlated with that of their corresponding miRNAs, which included 12 transcription factors, three plant hormone-related genes and one SUT. However, 22 targets showed a positive correlation with their corresponding miRNAs, including eight transcription factors, four plant hormone-related genes, one APL2 and one CTT (Figure 2 and Additional file 5). In total, there were 27 transcription factors and plant hormone-related genes, and three starch biosynthesis- and sugar transport-related genes among these target genes, this might be explained by translation suppression or feedback regulation. Surprisingly, some of the miRNAs slicing their targets were confirmed, but being positively correlated with the targets; these miRNA:target pairs included miR156: SPL13b, miR169:NF-YA3 and miR394:APL2/F-box.

On the basis of the miRNA:target pair confirmation and the co-expression analysis, 13 miRNA:target pairs were chosen for further assays of their regulatory relationship by quantitative real time PCR (qRT-PCR) in Arg7 storage roots at different growth stages; the corresponding primers are listed in Additional files 2 and 6. From the results, eight miRNAs and their corresponding targets had obvious negative correlations, except for miR160b; most of these miRNAs were expressed during the later stage of growth of the Arg7 root (Figure 3). For example, miR394 targets both cassava4.1_021267m (APL2) and cassava4.1_000867m (sucrose phosphate synthase $2 \mathrm{~F}, S P S 2 F$ ), which are involved in starch biosynthesis. APL2 showed low expression from 120 DAP (day after planting) to 180 DAP, increased from 180 DAP and reached a peak at 210 DAP; it then fell close to its minimum at 240 DAP. In contrast, an increase in levels of miR394 at 180 DAP, followed by a sustained increase from 210 DAP to 240 DAP, suggested that APL2 was negatively regulated by miR394 at a later stage. The second target, SPS2F, maintained a relatively stable expression level at the early stage, but then fell close to zero from 180 DAP to $240 \mathrm{DAP}$; this suggested that miR394 also negatively regulated SPS2F (Figure 3G). However, given that there was no negative/positive correlation between miR394 and APL2 on the basis of the RNA-seq data in roots among the three Manihot genotypes, the regulation might only have existed in a specific growth stage of cassava storage root.

\section{Discussion}

The initial findings about miRNA in cassava were obtained with the aid of the castor bean genome information; specifically, 20 conserved miRNA families were reported [22], and then 17 conserved miRNA families were predicted in 
Table 4 Confirmation of miRNA:target pairs by RLM-RACE

\begin{tabular}{|c|c|c|c|c|c|}
\hline \multirow[t]{2}{*}{ miRNA } & \multirow[t]{2}{*}{ Target } & \multirow[t]{2}{*}{ Annotation } & \multicolumn{3}{|l|}{ Cleavage sites } \\
\hline & & & Upstream & Complementary region & Downstream \\
\hline \multirow[t]{2}{*}{ miR156/miR535 } & cassava4.1_006419m & SPL2 & / & 10th/11th(8), 11th/12th(3) & $-35 \operatorname{th} /-36 \operatorname{th}(1)$ \\
\hline & cassava4.1_009657m & SPL13b & / & $\begin{array}{l}9 \operatorname{th} / 10 \operatorname{th}(2), 10 \text { th } / 11 \operatorname{th}(4), \\
11 \text { th/12th(1) }\end{array}$ & $-1 \mathrm{st} /-2 \mathrm{nd}(1)$ \\
\hline \multirow[t]{2}{*}{ miR159/miR319 } & cassava4.1_004606m & MYB33 & / & 11 th/12th(10) & $-52 \operatorname{th} /-53 \operatorname{th}(1),-115 \operatorname{th} /-116 \mathrm{th}(1)$ \\
\hline & cassava4.1_030321m & MYB81 & / & 11th/12th(6) & -59 th $/-60^{\text {th }}(1)$ \\
\hline \multirow[t]{2}{*}{$\operatorname{miR394}$} & cassava4.1_021267m & APL2 & $\begin{array}{l}+18 \operatorname{th}(1),+35 \operatorname{th}(3), \\
+42 \operatorname{th}(1),+69 \operatorname{th}(2)\end{array}$ & $2 \mathrm{nd}(3), 14 \operatorname{th}(1)$ & $\begin{array}{l}-27 \operatorname{th}(1),-29 \operatorname{th}(1),-49 \operatorname{th}(1),-92 \operatorname{th}(1), \\
-105 \operatorname{th}(1),-109 \operatorname{th}(1),-139 \operatorname{th}(1)\end{array}$ \\
\hline & cassava4.1_007038m & F-box & / & 10th(2) & $\begin{array}{l}-31 \operatorname{th}(1),-52 \operatorname{th}(1),-55 \operatorname{th}(1),-59 \operatorname{th}(1), \\
-68 \operatorname{th}(1),-75 \operatorname{th}(1),-102 \operatorname{th}(1)\end{array}$ \\
\hline $\operatorname{miR} 160$ & cassava4.1_002668m & ARF10 & / & $11^{\text {th }}(10)$ & / \\
\hline miR167 & cassava4.1_009942m & $R D 19$ & $\begin{array}{l}+5 \operatorname{th}(1),+25 \operatorname{th}(1) \\
+145 \operatorname{th}(1),+196 \operatorname{th}(1)\end{array}$ & & $-41 \operatorname{th}(1)$ \\
\hline $\operatorname{miR} 169$ & cassava4.1_011576m & NF-YA3 & $+30 \operatorname{th}(1),+6 \operatorname{th}(1)$ & 11th(5), 15th(1) & / \\
\hline miR396 & cassava4.1_003731m & GRF1 & $+69 \operatorname{th}(2)$ & $6 \operatorname{th}(1), 11$ th(6) & $-45 \operatorname{th}(1),-60 \operatorname{th}(1),-188 \operatorname{th}(1)$ \\
\hline miR398 & cassava4.1_024493m & DIR-like & $+8 \operatorname{th}(10)$ & / & / \\
\hline miR477 & cassava4.1_008074m & $C L B$ & $+37 \operatorname{th}(8)$ & / & / \\
\hline miR166 & cassava4.1_009671m & GH17 & / & / & $-8 \operatorname{th}(3),-16 \operatorname{th}(6),-34 \operatorname{th}(1)$ \\
\hline new-21 & cassava4.1_006393m & EDR2 & / & / & $\begin{array}{l}-112 \operatorname{th}(6),-160 \operatorname{th}(2),-176 \operatorname{th}(1) \\
-177 \operatorname{th}(1),-178 \operatorname{th}(1),-182 \operatorname{th}(1)\end{array}$ \\
\hline
\end{tabular}

Note: As an example, 10th/11th (8) indicate that there were eight cleavage sites at the 10th or 11th nucleotide in the complementary region of cassava4.1_006419m and miR156/535; alternatively, -35 th/-36th (1) indicates that there was one cleavage site downstream of the complementary region of cassava4.1_006419m and miR156/535.

cassava by using Arabidopsis mature miRNAs as seed sequences [23]. Recently, 169 potential conserved miRNAs in cassava were identified by a computational approach, and 126 miRNAs (114 conserved and 12 new) were discovered by small RNA sequencing $[19,20]$. In this study, we discovered 107 conserved miRNAs (29 families) and 39 new miRNAs ( 33 families) by small RNA sequencing, and 36 of these families were detected again by RT-PCR. By miRNA transcriptome profiling, we identified 41 conserved miRNAs (17 families) and 19 new miRNAs

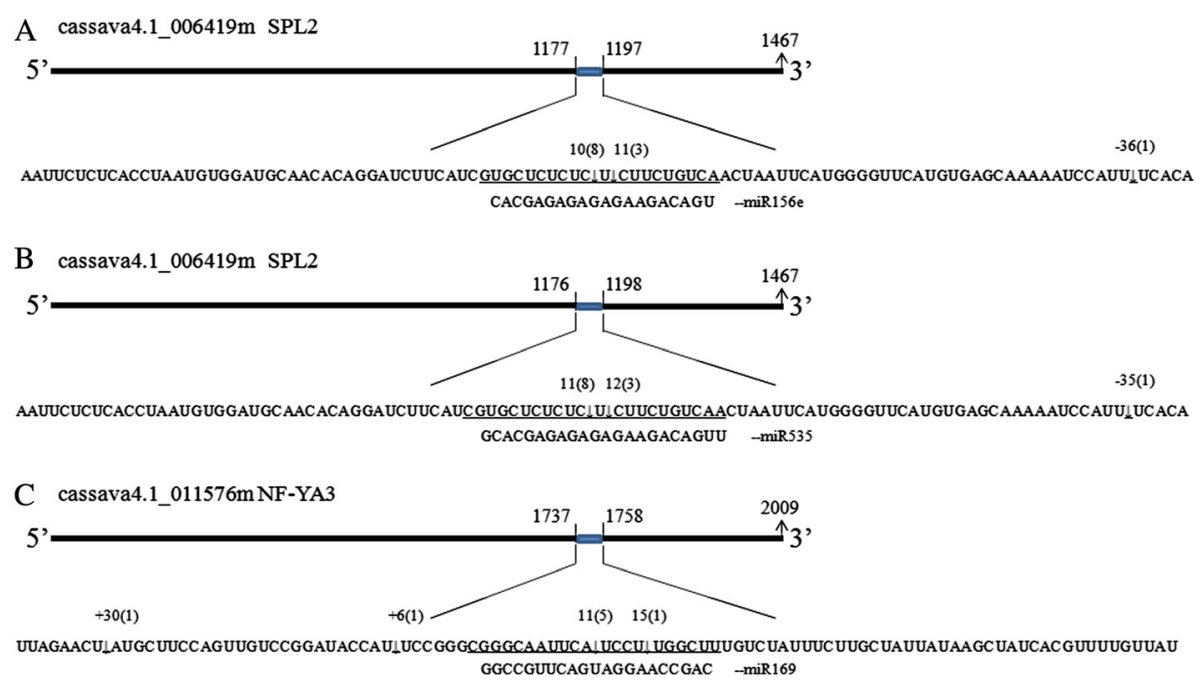

Figure 1 Identification of miRNA-guided cleavage products of target genes in cassava (partial results). The cleavage sites of selected targets as identified by 5' RACE analysis. For each miRNA, the target sequence is shown on the top and the miRNA sequence on the bottom. The numbers indicate the fraction of cloned PCR products when PCR was terminated at different positions. (A) The site of cleavage of cassava4.1_006419m by miR156. (B) The site of cleavage of cassava4.1_006419m by miR535. (C) The site of cleavage of cassava4.1_011576m by miR169d. 


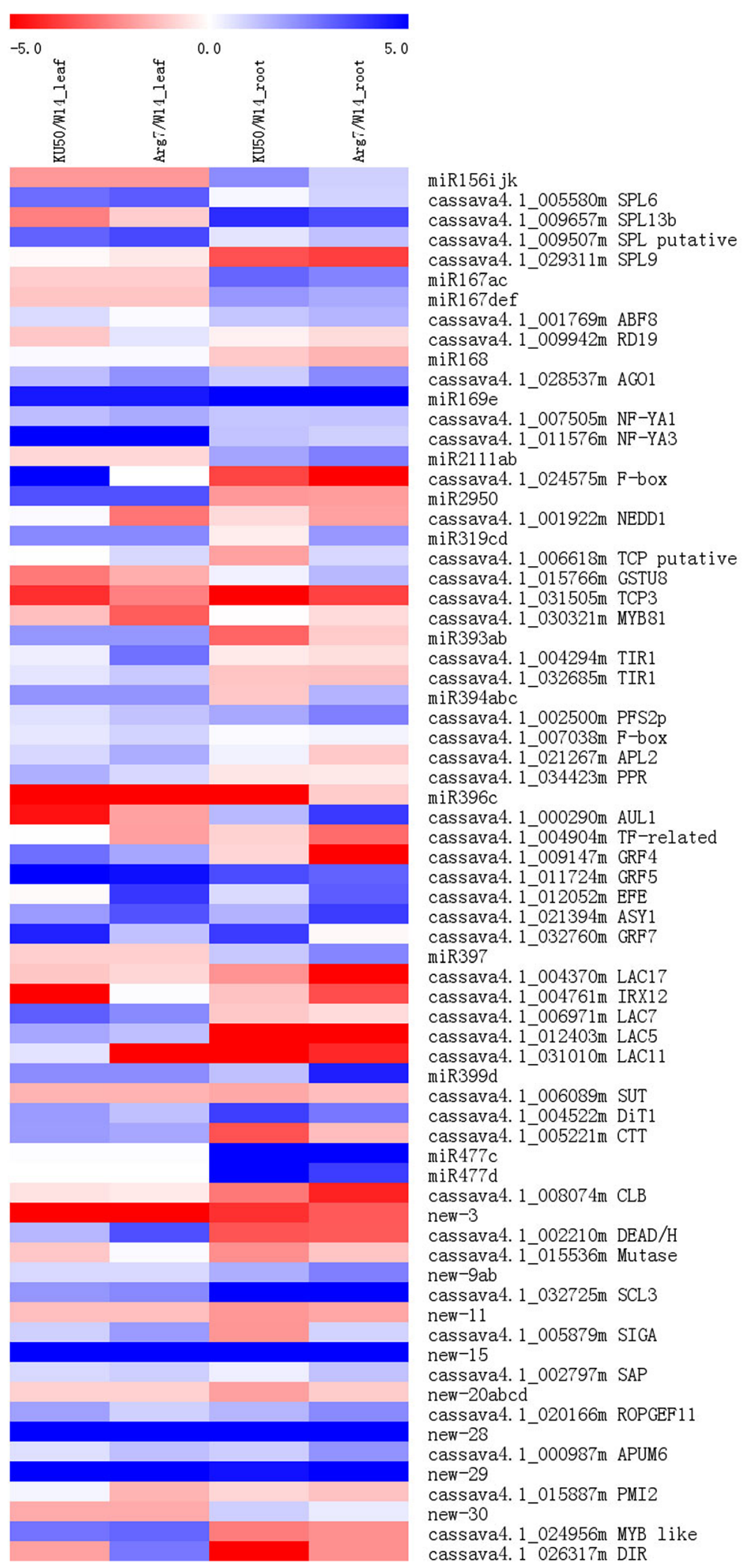

Figure 2 Correlations of the levels of expression of miRNAs and their corresponding targets in leaf and storage root between cultivars and their wild progenitor. Heat mapping was performed based on the log2- normalized expression ratio of cultivar/wild progenitor. 


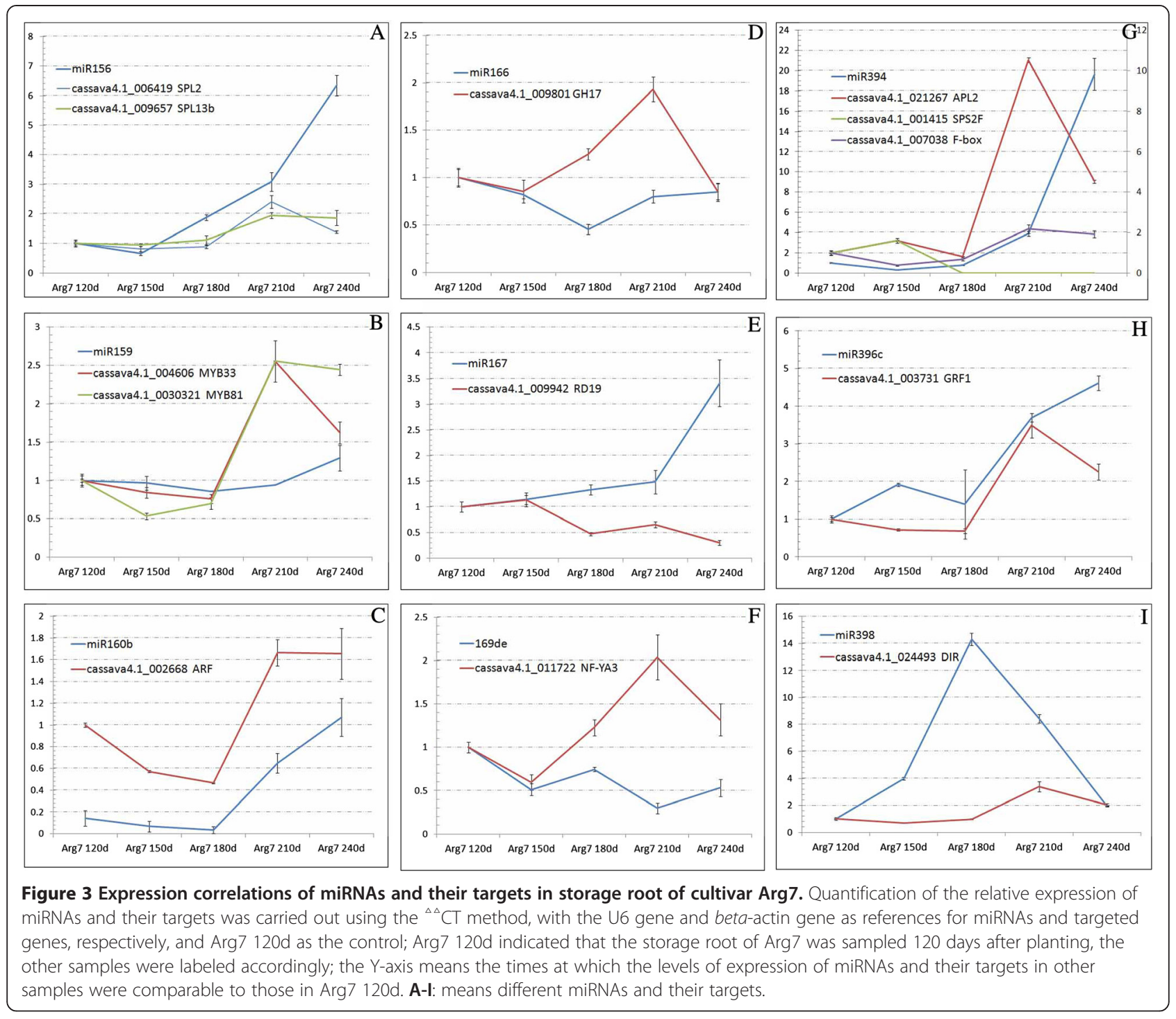

(15 families) that showed differential expression between the cultivars and their wild progenitor. Recently, Xia et al. also reported 22 cassava new miRNAs, and part of them responded to chilling stress [24].

We predicted that 360 cassava unigenes were targeted by 26 conserved and 27 new miRNA families, and that 57 of them (including $M Y B, S P L, A R F, N A C$ and TCP) encode transcription factors; several similar results have already been reported in cassava $[19,20]$ and other species $[5,25,26]$. Several miRNAs are involved in starch accumulation in rice [6], and five of them (miR159, miR160, miR164, miR167 and miR319) also had the same targets in cassava. In addition, two other miRNAs (miR394 and miR399) targeted APL2 and three sugar and carbohydrate metabolism-related genes (sugar transporter, invertase and carbohydrate transmembrane transporter), which have not been reported in other species. These miRNAs might be key regulators of starch biosynthesis in cassava.

A previous study reported that miR159 regulated $M Y B$ mRNAs, and miR319 predominantly acted on TCP mRNAs in Arabidopsis [27]. Both miR159 and miR319 shared sequence identity at 20 of 22 nucleotides, and the expression level of miR159 was far greater than that of miR319 in two cultivars and the wild progenitor. Therefore, similar to the case in Arabidopsis, MYB transcription factors were mainly regulated by miR159 in cassava. MYB could function as a transcriptional activator in ABA-inducible gene expression in Arabidopsis $[28,29]$, and high concentrations of ABA could suppress the expression of starch synthesis genes in maize and rice [30,31]. It was inferred that miR159 could directly or indirectly affect starch biosynthesis in cassava. 
miR396 targeted 20 cassava unigenes, including five GRF transcription factors. MeGRF1 (cassava4.1_003731) was verified to be sliced by miR396 in cassava. Previous studies reported that high expression level of miR396 in root tips might result in reduced expression of six $M t G R F$ genes [32], and that the miR396-GRF1/GRF3 regulatory module acted as a developmental regulator in the reprogramming of root cells during cyst nematode infection in Arabidopsis [33]. These findings suggested that miR396 might regulate root development in cassava.

miR169 targeted NF-YA family genes, and overexpression of NF-YA5 and NF-YA3 or down-regulation of miR169 might enhance drought stress tolerance in Arabidopsis and soybean [34,35]. MeNF-YA3 was negatively regulated by miR169, and the expression level of miR169 in the wild progenitor was lower than that in cultivars, and the evidence was that the wild progenitor had stronger drought tolerance than the cultivars usually.

miR398 targeted the mRNA of a disease resistanceresponsive family protein (cassava4.1_024493, Dir-like) in cassava, but the cleavage sites of the miR398:Dir-like pair were not positioned within the CR: ten cleavage sites were all at the 8th nucleotide upstream of the CR. Given that the miRNA-guided cleavage occurred quite precisely at the 10th or 11th nucleotide from the $5^{\prime}$ end of the miRNA in CR [24,36,37], this might be a surprising phenomenon that is difficult to explain.

\section{Conclusion}

Using next-generation sequencing technology, we carried out miRNA transcriptome and transcriptome profiling of two cultivated cassava and their wild progenitor. A total of 107 conserved miRNAs (29 families) and 39 new miRNAs (33 families) were identified, and most miRNAs were highly expressed in the cultivars. Of the 360 unigenes predicted to be the targets of 53 cassava miRNA families, 14 unigenes were confirmed. In addition, co-expression analysis between miRNAs and their targets was performed on the basis of the miRNA transcriptome and transcriptome profiling of leaves and storage roots; the expression levels of 28 targets were negatively correlated with that of their corresponding miRNAs. In conclusion, the differential expression of miRNAs between cultivars and their wild progenitor, together with our analysis of GO annotation and confirmation of miRNA:target pairs, might provide insight into how the wild progenitor was domesticated to cultivated cassava.

\section{Methods}

\section{Plant materials}

Two cultivars of the cultivated species Manihot esculenta Crantz (KU50 and Arg7) and W14, a subspecies of Manihot esculenta spp. flabellifolia, were used in this study. Both KU50 (a cultivar that is extensively planted in South East Asia) and Arg7 (a cultivar from Argentina) presented with higher photosynthesis and higher storage-root yield and starch content of storage roots. This distinguished them from W14, a native of Central Brazil, which had a lower rate of photosynthesis and very low storage root yield and starch content of the storage root. Manihot esculenta spp. flabellifolia was previously proposed to be the progenitor of cultivated cassava [38-40]. All three genotypes were grown in an experiment field in Haikou, China. Leaves and roots of these three genotypes were sampled at 150 DAP for sequencing of small RNAs and characterisation by RNA-seq. The roots of Arg7 were sampled on 120 DAP, 180 DAP, 210 DAP and 240 DAP for expression profile analysis of miRNA and targets by real-time PCR, and miRNA-target pair confirmation by RLM-RACE.

\section{Small RNA extraction and Solexa sequencing}

Small RNA samples of leaves and roots from the above three Manihot genotypes were extracted by using an miRNA isolation kit (Bioteke, Beijing, China) in accordance with the manufacturer's instructions. Small RNAs of fewer than 30 bases were isolated from these miRNA samples, and linked with a pair of Solexa adaptors to their 3' and 5' ends; then, the sample was reverse-transcribed into cDNA and amplified using the adaptor primers. The doublestranded miR-cDNAs were sequenced using Illumina's Solexa Sequencer in accordance with the manufacturer's instructions (BGI Company, Shenzhen, China).

\section{RNA extraction and sequencing}

Total RNA was extracted from leaves and storage roots using RNAplant reagent (Tiangen, Beijing, China) and purified using RNeasy Plant Mini Kit (Qiagen, Valencia, CA). The cDNA libraries for analysis using a Illumina Hiseq2000 instrument were prepared by following the protocol of Zhong et al. [41]. Six cDNA libraries of leaf and storage root were sequenced, and the sequenced reads were aligned to the cassava genome draft (http://www.phytozome.net/cassava) using TopHat and Cufflinks [42] and annotated using KEGG [43]. The fragments per kilobase per million reads (FPKMs) were used to normalise gene expression counts for each transcript. Transcripts with FPKM $<3$ were considered to be so rare as to not be expressed at all, as suggested for a study on white lupin [44].

\section{Identification of conserved and new miRNAs in wild and cultivated species}

1) Conserved miRNA Mature plant miRNA sequences from miRBase (http://www.mirbase.org/) were aligned to the AM560 genome (http://www.phytozome.net/ cassava). We retrieved the flanking genomic 
sequences around completely matched loci, with different upstream and downstream lengths, to form possible precursors of candidate miRNAs with the RNAfold program [45]. We chose those sequences with folding structures that have at least $18 \mathrm{bp}$ in matched regions, one central loop and a folding energy $\leq-18 \mathrm{kcal} / \mathrm{mol}$. The free tails in the secondary structures were then removed. Next, we applied the MiRcheck program [46] to select sequences that have $\leq 4$ mismatches, $\leq 2$ bulged or asymmetrically unpaired nucleotides and $\leq 2$ continuous mismatches in the seed regions.

2) New miRNA.

We searched for new miRNAs in the three Manihot genotypes (namely, W14, Arg7 and KU50), using the corresponding small RNA-seq datasets. The newly identified miRNAs, combined with known miRNAs in Manihot, were then subjected to a homology search. We aligned mature and hairpin sequences of an miRNA to the cultivar-AM560 genome using the local alignment tool BLAST. We set the p-value obtained from BLAST to less than $1 \mathrm{e}-10$ and manually examined the alignment to determine whether a BLAST hit was homologous to the input miRNA. We mapped the qualified reads from corresponding cultivar datasets to the identified homologous sequence using Bowtie [47] and counted the mappable reads. If no homologous sequences could be identified in a cultivar genome assembly, we then mapped reads from the sequencing datasets of the same cultivar to the input miRNA sequence, allowing two mismatches. We considered a miRNA to be not conserved in the cultivar genome assembly if both of the following criteria were met: 1) no homologous sequences identified and 2) insufficient mappable reads (fewer than 10 normalised reads) for analysis. If any of the reads mapped to the input miRNA sequences, we considered the miRNA to be conserved in the genome assembly even if we were not able to identify the homologous sequences.

\section{Identification of differentially expressed miRNAs}

Reads that aligned perfectly to the candidate miRNAyielding transcripts were used to compute the digital expression levels of the miRNAs. Reads that mapped to multiple genomic loci were attributed to all derivative miRNAs. Read counts in each sample were normalised to adjust for sample variation. If $N_{\text {sample }}$ is the number of qualified reads that aligned to the genome and cDNA sequences in that sample and $C$ is the average value of $N_{\text {sample }}$ of all samples, then the NNR for each miRNA in each sample is $\left(N_{\text {miRNA }}{ }^{*} C / N_{\text {sample }}\right)$, where $N_{\text {miRNA }}$ is the raw sequencing reads of the miRNA. Differentially expressed miRNAs were those that had at least two fold changes between the cultivars and their wild progenitor.
Target mRNA prediction and miRNA: target pair validation

We used the Hitsensor algorithm [48] to predict miRNA targets in AM560, which was downloaded from http://www. phytozome.net/cassava. Hitsensor searched for miRNA complementary sites in coding regions with a modified Smith-Waterman algorithm [49]. This algorithm scores these sites by giving rewards to key sequence-specific determinants, including the seed region (12-17-nucleotides long), local-AU content around the seed region and $\leq 3$ mismatches.

RLM-RACE Gene Racer Kit (Invitrogen, CA, USA) was used to validate the predicted interaction. An RNA adaptor was ligated to the truncated mRNA, followed by reverse transcription with polyT. The next nested PCR with two gene-specific primers and two GeneRacer 5' forward primers was performed as described previously [22]. The amplified PCR products were gel-purified, cloned into the PMD-18T vector (Takara, Dalian, China) and sequenced.

\section{Quantity detection of miRNA and targets}

The amount of miRNA was quantified as described previously [50]. Firstly, the small RNA samples were converted to miR-cDNA by using an RT primer pool with reverse transcriptase. Then, a specific primer pair was designed for each miRNA, after which PCR amplification with SYBR Premix Ex TaqTM kit (Takara, Dalian, China) was carried out using a Rotor-Gene 6000 machine (Corbett Robotics, Australia), with the U6 gene as a control. Quantification of the relative expression of miRNAs was performed using the ${ }^{\Delta \Delta} \mathrm{CT}$ method. Quantification of the target was also carried out using qRT-PCR, with the beta-actin gene as a control. The forward and reverse primers of the target were located at the two flanks of the binding region at which the miRNA interacted with its target mRNA; the primer pairs are listed in Additional file 6.

\section{Supporting data}

The small RNA sequencing data is deposited in Gene omnibus with accession number GSE52178. RNA-seq reads are deposited in GenBank/SRR sequence read archive under the accession codes SRR1299000, SRR1299003, SRR1299009, SRR1299006, SRR1298998 and SRR1298996.

\section{Additional files}

Additional file 1: Normalised digital read counts for each miRNA in leaf and storage root of three Manihot genotypes.

Additional file 2: Primer sets used to amplify microRNAs using qRT-PCR.

Additional file 3: Functional annotation of 360 predicted targeted genes.

Additional file 4: Gene-specific primers for target validation by RLM-RACE. 
Additional file 5: Expression correlations of 21 miRNAs and their corresponding targets in leaf and root between cultivar and progenitor. "\#" means that the slicing is confirmed by RLM-RACE between miRNA and target.

Additional file 6: Primer pairs used to amplify targets for analysis by quantitative real-time PCR.

\section{Abbreviations}

DAP: Day after planting; miRNA: MicroRNA; GRT-PCR: Quantitative reverse transcription polymerase chain reaction; NGS: Next-generation sequencing; NNR: Normalised number of reads; FPKM: Fragment per kilobase per million reads.

\section{Competing interests}

The authors declare that they have no competing interests.

\section{Authors' contributions}

XC prepared the manuscript. XC and CZ performed miRNA:target pair validation and GRT-PCR analysis. JX and ZX participated in miRNA prediction, the expression profiling of miRNA targets and the characterisation of miRNA targets. $\mathrm{HZ}$ and $\mathrm{CL}$ were involved in preparing the plant materials and deep sequencing, WW and WZ designed the project. All authors read and approved the final manuscript.

\section{Acknowledgements}

This work was supported financially by the National Basic Research and Development Program (2010CB126600), Natural Science Foundation of China (31101193) and the earmarked fund for China Agriculture Research System (CARS-12).

\section{Author details}

'The Institute of Tropical Bioscience and Biotechnology (ITBB), Chinese Academy of Tropical Agricultural Sciences (CATAS), Haikou 571101, PR China. ${ }^{2}$ Key Laboratory of Biology and Genetic Resources of Tropical Crops, Ministry of Agriculture, Haikou 571101, PR China. Institute for Systems Biology, Jianghan University, Wuhan 430056, China. ${ }^{4}$ Department of Computer Science and Engineering, Washington University in St. Louis, St. Louis, Missouri, MO 63130, USA.

Received: 23 June 2014 Accepted: 27 November 2014 Published online: 04 February 2015

\section{References}

1. Lagos-Quintana M, Rauhut R, Lendeckel W, Tuschl T: Identification of novel genes coding for small expressed RNAs. Science 2001, 294(5543):853-858.

2. Lau NC, Lim LP, Weinstein EG, Bartel DP: An abundant class of tiny RNAs with probable regulatory roles in Caenorhabditis elegans. Science 2001, 294(5543):858-862.

3. Chen X: MicroRNA biogenesis and function in plants. FEBS Lett 2005, 579(26):5923-5931

4. Carla S, Edgardo GB, Silvana VS, Javier FP: Role of MicroRNA miR319 in Plant Development, in R. Sunkar (ed.), MicroRNAs in Plant Development and Stress Responses, Signaling and Communication in Plants 15, doi:10.1007/978-3-64227384-1, Springer-Verlag Berlin Heidelberg 2012.

5. Ozhuner E, Eldem V, Ipek A, Okay S, Sakcali S, Zhang B, Boke H, Unver T: Boron stress responsive miRNAs and their targets in barley. PLoS One 2013, 8(3):e59543.

6. Peng T, Sun H, Du Y, Zhang J, Li J, Liu Y, Zhao Y, Zhao Q: Characterization and expression patterns of miRNAs involved in rice grain filling. PLoS One 2013, 8(1):e54148

7. Chen XB, Zhang ZL, Liu DM, Zhang K, Li AL, Mao L: SQUAMOSA promoter-binding protein-like transcription factors: star players for plant growth and development. J Integr Plant Biol 2010, 52(11):946-951.

8. Park W, Li J, Song R, Messing J, Chen X: CARPEL FACTORY, a Dicer homolog, and HEN1, a novel protein, act in miRNA metabolism in Arabidopsis thaliana. Curr Biol 2002, 12:1484-1495.

9. Palatnik JF, Allen E, Wu X, Schommer C, Schwab R, Carrington JC, Weigel D: Control of leaf morphogenesis by miRNAs. Nature 2003, 425(6955):257-263.
10. Aukerman MJ, Sakai H: Regulation of flowering time and floral organ identity by a MiRNA and its APETALA2-like target genes. Plant Cell 2003, 15(11):2730-2741.

11. Sunkar R, Zhu JK: Novel and stress-regulated miRNAs and other small RNAs from Arabidopsis. Plant Cell 2004, 16(8):2001-2019.

12. Sun G: MicroRNAs and their diverse functions in plants. Plant Mol Biol 2012, 80(1):17-36.

13. Reinhart BJ, Weinstein EG, Jones-Rhoades MW, Bartel B, Bartel DP: MiRNAs in plants. Genes Dev 2002, 16:1616-1626.

14. Sunkar R, Jagadeeswaran $\mathrm{G}$ : In silico identification of conserved miRNAs in large number of diverse plant species. BMC Plant Biol 2008, 8:37.

15. Guo W, Wu G, Yan F, Lu Y, Zheng H, Lin L, Chen H, Chen J: Identification of novel Oryza sativa miRNAs in deep sequencing-based small RNA libraries of rice infected with rice stripe virus. PLoS One 2012, 7(10):e46443.

16. Tetlow IJ, Morell MK, Emes MJ: Recent developments in understanding the regulation of starch metabolism in higher plants. J Exp Bot 2004, 55(406):2131-2145.

17. Xie F, Frazier TP, Zhang B: Identification and characterization of microRNAs and their targets in the bioenergy plant switchgrass (Panicum virgatum). Planta 2010, 232(2):417-434.

18. Xie F, Frazier TP, Zhang B: Identification, characterization and expression analysis of MicroRNAs and their targets in the potato (Solanum tuberosum). Gene 2011, 473(1):8-22.

19. Patanun O, Lertpanyasampatha M, Sojikul P, Viboonjun U, Narangajavana J: Computational identification of miRNAs and their targets in cassava (Manihot esculenta Crantz). Mol Biotechnol 2013, 53(3):257-269.

20. Pérez-Quintero ÁL, Quintero A, Urrego O, Vanegas P, López C: Bioinformatic identification of cassava miRNAs differentially expressed in response to infection by Xanthomonas axonopodis pv. manihotis. BMC Plant Biol 2012, 12:29.

21. Prochnik S, Marri PR, Desany B, Rabinowicz PD, Kodira C, Mohiuddin M, Rodriguez F, Fauquet C, Tohme J, Harkins T, Rokhsar DS, Rounsley S: The cassava genome: current progress, future directions. Trop Plant Biol 2012, 5(1):88-94.

22. Zeng $C Y$, Wang WQ, Zheng $Y$, Chen $X$, Bo WP, Song S, Zhang WX, Peng $M$ : Conservation and divergence of miRNAs and their functions in Euphorbiaceous plants. Nucle Acid Res 2010, 38:981-995.

23. Amiteye S, Corra JM, Sharbe TF: Overview of the potential of microRNAs and their target gene detection for cassava (Manihot esculenta) improvement. Afr J Biotech 2011, 10(14):2562-2573.

24. Xia J, Zeng CY, Chen Z, Zhang K, Chen X, Zhou YF, Song S, Lu C, Yang RJ, Yang Z, Zhou J, Peng H, Wang WQ, Peng M, Zhang WX: Endogenous small-noncoding RNAs and their roles in chilling response and stress acclimation in Cassava. BMC Genomics 2014, 15:634.

25. Karlova R, van Haarst JC, Maliepaard C, van de Geest H, Bovy AG, Lammers M, Angenent GC, de Maagd RA: Identification of miRNA targets in tomato fruit development using high-throughput sequencing and degradome analysis. J Exp Bot 2013, 64(7):1863-1878.

26. Carnavale Bottino M, Rosario S, Grativol C, Thiebaut F, Rojas CA, Farrineli L, Hemerly AS, Ferreira PC: High-throughput sequencing of small RNA transcriptome reveals salt stress regulated miRNAs in sugarcane. PLoS One 2013, 8(3):e59423.

27. Palatnik JF, Wollmann H, Schommer C, Schwab R, Boisbouvier J, Rodriguez R, Warthmann N, Allen E, Dezulian T, Huson D, Carrington JC, Weigel D: Sequence and expression differences underlie functional specialization of Arabidopsis microRNAs miR159 and miR319. Dev Cell 2007, 13(1):115-125.

28. Abe H, Urao T, Ito T, Seki M, Shinozaki K, Yamaguchi-Shinozaki K: Arabidopsis AtMYC2 (bHLH) and AtMYB2 (MYB) function as transcriptional activators in abscisic acid signaling. Plant Cell 2003, 15(1):63-78.

29. Reyes $\mathrm{J}$, Chua NH: ABA induction of miR159 controls transcript levels of two MYB factors during Arabidopsis seed germination. Plant $J$ 2007, 49(4):592-606.

30. Chen J, Huang B, Li Y, Du H, Gu Y, Liu H, Zhang J, Huang Y: Synergistic influence of sucrose and abscisic acid on the genes involved in starch synthesis in maize endosperm. Carbohydr Res 2011, 346(13):1684-1691.

31. Zhu GH, Ye NH, Yang JC, Peng XX, Zhang JH: Regulation of expression of starch synthesis genes by ethylene and $A B A$ in relation to the development of rice inferior and superior spikelets. J Exp Bot 2011, 62(11):3907-3916.

32. Bazin J, Khan GA, Combier JP, Bustos-Sanmamed P, Debernardi JM, Rodriguez $R$, Sorin C, Palatnik J, Hartmann C, Crespi M, Lelandais-Brière C: MiR396 affects 
mycorrhization and root meristem activity in the legume Medicago truncatula. Plant J 2013, 74(6):920-934.

33. Hewezi T, Maier TR, Nettleton D, Baum TJ: The Arabidopsis miRNA396-GRF1/GRF3 regulatory module acts as a developmental regulator in the reprogramming of root cells during cyst nematode infection. Plant Physiol 2012, 159(1):321-335.

34. Li WX, Oono Y, Zhu J, He XJ, Wu JM, lida K, Lu XY, Cui X, Jin H, Zhu JK: The Arabidopsis NFYA5 transcription factor is regulated transcriptionally and post-transcriptionally to promote drought resistance. Plant Cell 2008, 20(8):2238-2251

35. Ni Z, Hu Z, Jiang Q, Zhang H: GmNFYA3, a target gene of miR169, is a positive regulator of plant tolerance to drought stress. Plant Mol Biol 2013, 82(1-2):113-129.

36. Carra A, Mica E, Gambino G, Pindo M, Moser C, Pè ME, Schubert A: Cloning and characterization of small non-coding RNAs from grape. Plant J 2009, 59(5):750-763

37. Beauclair $L, Y u$ A, Bouché N: miRNA-directed cleavage and translational repression of the copper chaperone for superoxide dismutase mRNA in Arabidopsis. Plant J 2010, 62(3):454-462.

38. Carvalho Luiz Joaquim CB, Schaal BA: Assessing genetic diversity in the cassava (Manihot esculenta Crantz) germplasm collection in Brazil using PCR-based markers. Euphytica 2001, 120(1):133-142.

39. Olsen KM, Schaal BA: Microsatellite variation in cassava (Manihot esculenta, Euphorbiaceae) and its wild relatives: further evidence for a southern Amazonian origin of domestication. Am J Bot 2001, 88(1):131-142.

40. Léa M, Doyle M, Gilda SM, Bruno C, Nick PR: The evolutionary fate of phenotypic plasticity and functional traits under domestication in manioc: changes in stem iomechanics and the appearance of stem brittleness. PLoS One 2013, 8(9):1-20.

41. Zhong SL, Joung JG, Zheng Y, Chen YR, Liu B, Shao Y, Xiang JZ, Fei ZJ, Giovannoni JJ: High-throughput illumina strand-specific RNA sequencing library preparation. Cold Spring Harb Protoc 2011, 2011(8):940-949.

42. Trapnell C, Roberts A, Goff L, Pertea G, Kim D, Kelley DR, Pimentel H, Salzberg SL, Rinn JL, Pachter L: Differential gene and transcript expression analysis of RNA-seq experiments with TopHat and Cufflinks. Nat Protoc 2012, 7(3):562-578

43. Kanehisa M, Goto S, Kawashima S, Okuno Y, Hattori M: The KEGG resource for deciphering the genome. Nucle Acid Res 2004, 32:277-280.

44. O'Rourke JA, Yang SS, Miller SS, Bucciarelli B, Liu J, Rydeen A, Bozsoki Z, Uhde-Stone C, Tu ZJ, Allan D, Gronwald JW, Vance CP: An RNA-Seq transcriptome analysis of orthophosphate-deficient white lupin reveals novel insights into phosphorus acclimation in plants. Plant Physiol 2013, 161(2):705-724.

45. Hofacker IL: Vienna RNA secondary structure server. Nucle Acid Res 2003, 31:3429-3431.

46. Rhoades MW, Reinhart BJ, Lim LP, Burge CB, Bartel B, Bartel DP: Prediction of plant microRNA targets. Cell 2002, 110(4):513-520.

47. Langmead B, Trapnell C, Pop M, Salzberg SL: Ultrafast and memory-efficient alignment of short DNA sequences to the human genome. Genome Biol 2009, 10(3):R25.

48. Zheng $Y$, Zhang $W$ : Animal microRNA target prediction using diverse sequence-specific determinants. J Bioinform Comput Biol 2010, 8(4):763-788.

49. Smith TF, Waterman MS: Identification of common molecular subsequences. J Mol Biol 1981, 147:195-197.

50. Wang XW: A PCR based platform for miRNA expression profiling studies RNA 2009, 15:716-723.

\section{Submit your next manuscript to BioMed Central and take full advantage of:}

- Convenient online submission

- Thorough peer review

- No space constraints or color figure charges

- Immediate publication on acceptance

- Inclusion in PubMed, CAS, Scopus and Google Scholar

- Research which is freely available for redistribution 